\title{
The role of antimicrobial peptides in chronic inflammatory skin diseases
}

\author{
Małgorzata Marcinkiewicz, Sławomir Majewski \\ Department of Dermatology and Venereology, Medical University of Warsaw, Warsaw, Poland \\ Adv Dermatol Allergol 2016; XXXIII (1): 6-12 \\ DOI: $10.5114 /$ pdia.2015.48066
}

\begin{abstract}
Antimicrobial peptides (AMPs) are effector molecules of the innate immune system of the skin. They present an activity against a broad spectrum of Gram-positive and Gram-negative bacteria as well as some fungi, parasites and enveloped viruses. Several inflammatory skin diseases including psoriasis, atopic dermatitis, acne vulgaris and rosacea are characterized by a dysregulated expression of AMPs. Antimicrobial peptides are excessively produced in lesional psoriatic scales or rosacea in contrast to the atopic skin that shows lower AMP levels when compared with psoriasis. The importance of the AMPs contribution to host immunity is indisputable as alterations in the antimicrobial peptide expression have been associated with various pathologic processes. This review discusses the biology and clinical relevance of antimicrobial peptides expressed in the skin and their role in the pathogenesis of inflammatory skin diseases.
\end{abstract}

Key words: antimicrobial peptides, defensin, cathelicidin, LL-37, ribonuclease, innate immunity, inflammatory skin diseases, psoriasis.

\section{Introduction}

Human body is constantly exposed to various microbial and environmental agents. Skin and its protective mechanisms act as the first line of defense against invading pathogens. The epidermis of the skin forms not only a physical barrier impermeable to most infectious organisms, but also provides an immediate response which enables human organisms to resist infection. The prompt response is mediated, inter alia, by low molecular weight proteins named antimicrobial peptides (AMPs) due to their properties. They are a crucial part of the natural innate immune system. The defense mechanisms of innate immunity detect and destroy microorganisms within minutes or hours in a non-specific manner, discriminating however very effectively between host cells and pathogens.

Antimicrobial peptides probably represent one of the first evolved mechanisms, providing initial defense and also contributing to the induction of adaptive immune responses. Antimicrobial peptides are known for their cutaneous antimicrobial and immunomodulatory properties and demonstrate the ability to inhibit not only bacterial, protozoal and fungal, but also viral infections (Table 1) [1-4]. The family of AMPs consists of, among others, $\alpha$ - and $\beta$-defensins, cathelicidins, S100 proteins and ribonucleases. These small, mostly cationic endogenous proteins are found in keratinocytes, eccrine gland cells, mast cells, phagocytes and sebocytes [5, 6]. Antimicrobial peptides are amphipathic, which enables them to interact with and disrupt lipid membranes, leading to cell lysis and death. Due to their cationic properties, they interact preferentially with negatively charged bacterial membranes rather than with neutrally charged mammalian cell membranes.

Some of AMPs are constitutively expressed in the skin, whereas some are induced upon contact with pathogens. The production of these peptides can be influenced not only by microorganisms themselves, but also by pro-inflammatory cytokines or tumor necrosis factor (TNF)- $\alpha$ [7]. Over the past two decades numerous studies have shown indisputably the role of several AMPs in chronic inflammatory skin diseases such as psoriasis vulgaris and atopic dermatitis (AD) [8]. Despite the impaired skin barrier which facilitates potentially pathogenic microbes to colonize the epidermis, patients with psoriasis surprisingly present a low frequency of skin infections, whereas patients with $A D$ are predominantly susceptible to particular cutaneous bacterial, fungal and viral infections. One possible explanation of the fact is the difference in the expression of AMPs.

Address for correspondence: Małgorzata Marcinkiewicz MD, Department of Dermatology and Venereology, Medical University of Warsaw, 82a Koszykowa St, 02-008 Warsaw, Poland, phone: +48 225012 13 13, e-mail: malgorzata_sochacka@gazeta.pl Received: 1.10.2014, accepted: 12.11.2014. 
Table 1 . The activity of selected AMPs in the skin

\begin{tabular}{ll}
\hline AMP & Activity \\
\hline hBD-2 & Killing activity preferentially against Gram-negative bacteria like E. coli, Enterococcus faecalis, \\
& Pseudomonas aeruginosa, Propionibacterium acnes \\
& Induced by HPV \\
& Chemotactic activity on T cells and dendritic cells \\
& Involved in wound repair \\
\hline hBD-3 & Broad spectrum against Gram-negative and Gram-positive bacteria including MRSA, vancomycin- \\
& resistant Enterococcus faecium (VRE) \\
& Induced by HPV \\
& Chemotactic activity on dendritic cells, T cells and monocytes \\
& Promotion of wound healing \\
\hline RNase 7 & Antimicrobial activity against Gram-negative bacteria (E. coli and P. aeruginosa) and Gram-positive \\
& bacteria (P. acnes, S. aureus, MRSA) and yeast (Candida albicans) \\
& Ribonucleolytic activity \\
\hline Psoriasin (S100A7) & Active against E. coli, P. acnes (enhanced activity in synergy with LL-37) \\
& Chemotactic for CD4+ T lymphocytes and neutrophils \\
\hline Human cathelicidin LL-37 & Antimicrobial (e.g. against E. coli, P. aeruginosa, S. aureus, E. faecalis, Klebsiella pneumoniae, P. acnes), \\
& antifungal (C. albicans) and antiviral (HIV-1) activity \\
& Chemotactic activity for neutrophils, monocytes and T cells \\
& Mediator of inflammation \\
& Promotion of re-epithelialization of healing skin and angiogenesis \\
\hline Dermcidin & Active against E. coli, E. faecalis, S. aureus, and C. albicans \\
\hline
\end{tabular}

This review will concentrate mostly on the biological and clinical relevance of well-studied classes of AMPs found in the human skin, though several less extensively studied antimicrobial proteins and peptides are known. The importance of the epithelial contribution to host immunity is evident as alterations in the antimicrobial peptide expression have been associated with various pathologic processes.

\section{Defensins}

Defensins are one of widely characterized groups of antimicrobial peptides in vertebrates. They are small cystine rich molecules with $\beta$-hairpin structure stabilized by conserved three-disulfide bonds $[9,10]$. Three subfamilies have been described: $\alpha$-, $\beta$-, and circular $\theta$-defensins [11]. The human $\alpha$-defensins -1 to -4 , also called human neutrophil peptides (HNP1, HNP2, HNP3 and HNP4), were originally identified in neutrophils [12]. On the contrary, human $\alpha$-defensins, HD-5 and HD-6, are enteric peptides predominantly expressed in Paneth cells.

First human $\beta$-defensins were discovered in the last decade of the $20^{\text {th }}$ century [13]. By now gene-based research has predicted 28 human $\beta$-defensins [14], however, only the first four peptides are extensively reported. Human $\beta$-defensin 1 (hBD-1) is constitutively expressed by epithelial cells mostly of the respiratory and urinary tissues [5], whereas hBD-2 and hBD-3 in keratinocytes present low levels at the steady stage and are typically inducible [9, 15-17]. In the normal skin, hBD-2 and hBD-3 expression is limited to the uppermost layers of the epidermis and/or stratum corneum. Human BD-2 and - 3 are upregulated in various epithelia including epithelium of respiratory, gastrointestinal, and genitor-urinary tracts upon stimulation by microorganisms and microbial components, such as lipopolysaccharides (LPS), and by pro-inflammatory cytokines. Human BD-2 and -3 are inducible by UVB irradiation as well [18]. Interestingly, the hBD-2 and -3 proteins have been isolated from psoriatic scales $[13,16]$.

Human defensins have a broad spectrum of antimicrobial activity. They are highly effective in killing Gram-negative bacteria like Escherichia coli and Pseudomonas aeruginosa and act against Candida albicans. Unlike hBD-1 and hBD-2, hBD-3 is bactericidal toward multiresistant Gram-positive Staphylococcus aureus and at low concentrations kills many other potential pathogenic microorganisms including Streptococcus pyogenes and vancomycin-resistant Enterococcus faecium [16]. Defensins have been considered pro-inflammatory as they stimulate keratinocytes to produce pro-inflammatory cytokines and chemokines such as interleukin (IL)-6, IL-10, monocyte chemoattractant protein-1 and macrophage inflammatory protein-3 $\alpha$ and mediate keratinocytes migration and proliferation [19]. Furthermore, their expression increases in response to TLR ligands, TNF- $\alpha$, IL- $1 \beta$, interferon (IFN) $-\gamma$ $[13,20]$. Additionally, hBD-2 recruits immature dendritic cells and attracts memory T cells through the CCR6 chemokine receptor linking innate and adaptive immune responses [21]. Ferris et al. also suggested an important role for hBD3 in inducing dendritic cell activation, migration, and polarization [22]. Recent studies point out to the immunosuppressive role of hBD-3 attenuating inflammatory 
cytokine responses. Importantly, defensins are able to neutralize transformed or cancerous cells, and this cytotoxicity tends to be neither species-specific nor selective [23]. Moreover, studies reported that defensins stimulate migration and proliferation of epidermal keratinocytes and thus might promote cutaneous wound healing [19].

\section{Cathelicidins}

Cathelicidins were first identified in 1995 [24]. Active cathelicidin peptides are cleaved from the inactive precursor by enzymatic processing [25]. To date only one cathelicidin has been found in a man. Encoded by the gene CAMP, human cathelicidin LL-37 is a multipotent peptide disrupting both bacterial membranes and viral envelopes, having anti-fungal activity against yeasts. The precursor of LL-37 is expressed by several cell types in the skin such as keratinocytes, neutrophils, eccrine ducts, and mast cells [26]. LL-37, a 37 amino acid long peptide, gains its activity like other cathelicidins after cleavage from a pro-peptide. In the healthy skin, its expression is barely detectable in keratinocytes. However, upregulation of LL-37 occurs after injury, infection or inflammation [27]. Its role in cutaneous wound healing was proven as well. Additionally, LL-37 presents a pro-apoptotic activity in airway epithelial cells, promotes differentiation of monocyte-derived DCs and it can stimulate angiogenesis through an increase in endothelial cell proliferation and vessel formation [28]. Apart from antimicrobial properties, human cathelicidin presents a receptor-mediated chemotactic activity and induction of neovascularization known as "alarmin" functions of LL-37. Activation of immune cells occurs via the formyl-peptide-like receptor-1 (FPRL-1), G-protein-coupled receptors, the nucleotide receptor P2X7 and TLR signaling [28-30].

\section{S100A family}

Psoriasin (S100A7), a Ca²+ binding S100 protein, was discovered in psoriatic lesions [31]. It is expressed at low levels in normal epithelial cells, but can be induced in keratinocytes under certain conditions [32]. The focal expression of psoriasin is found in the skin, especially in localizations associated with high density of bacteria. In addition, the peptide accumulates in the epidermis of sebaceous skin as well as sebaceous glands and is secreted to the external skin surface [33]. Psoriasin is associated with altered keratinocyte differentiation and has been found to be related to tumorigenesis [32]. It exhibits an antibacterial activity preferentially against $E$. coli. Apart from the antimicrobial activity, psoriasin is a potent and selective chemotactic inflammatory protein for CD4+ T lymphocytes and neutrophils [34].

Another member of the S100A family with an antimicrobial activity is calprotectin, a heterocomplex of the two calcium-binding proteins S100A8 and S100A9. Cal- protectin exerts antibacterial properties inter alia against E. coli, Klebsiella spp., S. aureus and Staphylococcus epidermidis, as well as fungistatic activity toward the fungus C. albicans [35]. The hypothesis has been raised that the complex may play a role in inflammatory skin diseases. In the epidermis, S100A8 and S100A9 are up-regulated in hyperproliferative keratinocytes like those in wound healing [36] and in psoriatic lesions [37].

\section{RNase 7}

RNase 7 belongs to the RNase A superfamily characterized by a lysine in the active center catalyzing the ribonuclease activity [38]. RNase 7 was extracted from skin-derived stratum corneum while searching for the presence of AMPs by Harder et al. and concurrently identified by the Zhang group [39, 40]. Peptide presents in vitro an antimicrobial activity against Gram-negative and Gram-positive bacteria, including E. coli, Pseudomonas aeruginosa, S. aureus as well as MRSA, E. faecium and its vancomycin-resistant strain, Propionibacterium acnes and against yeast $C$. albicans independent of RNase activity [33, 39, 41]. Simanski et al. postulated that RNase 7 is secreted on the skin surface and functions primarily outside the cell enabling the control of the growth of microorganisms on the skin surface [38].

\section{Dermcidin}

Dermcidin is one of the antimicrobial peptides that present an anionic structure. Dermcidin is proteolytically cleaved, generating dermcidin 1 (DCD-1). It is predominantly and constitutively expressed in eccrine sweat glands and released with sweat to the epidermal surface [6]. Thus, dermcidin exhibits an antimicrobial activity against a variety of pathogenic microorganisms like E. coli, Enterococcus faecalis, S. aureus and C. albicans under in vitro conditions [42].

\section{Clinical relevance of AMPs in inflammatory skin disorders}

The observation that antimicrobial peptides are expressed in the uppermost parts of the epidermis may suggest a protective function of these effector molecules of the innate immune system. They create a constitutive antimicrobial skin barrier composed of peptides like the S100 protein psoriasin and RNase 7, abundant on the skin surface. Alongside with psoriasin and RNase 7, skin surface is rich in dermcidin and lactoferrin released from sweat glands [43]. Another defense strategy is an additional barrier consisting of induced AMPs. Activated keratinocytes initiate the increase in antimicrobial peptides, including hBD-2, hBD-3 and cathelicidin LL-37. Furthermore, already expressed AMPs, especially psoriasin and RNase 7, are additionally induced by microorganisms [43]. 
It is tempting to say that there is an inverse correlation between severity of the inflammatory skin disease and the level of AMPs production. To support this hypothesis many studies concentrated on patients with AD. It has been hypothesized that increased susceptibility of $A D$ patients to superinfections with $S$. aureus may arise from the impaired expression of AMPs. Researches revealed a lower expression of hBD-2, hBD-3 and LL-37 in the skin of patients with $A D$ when compared to psoriasis vulgaris $[44,45]$. A possible explanation could be the lack of major inducers in the AD skin, such as IL-1, IL-17 and IL-22, alongside with inhibition of AMPs induction by an elevated level of Th2 cytokines (IL-4, IL-10 and IL-13) [46]. However, the observation of AMPs in AD was based on the comparison to the lesional psoriatic skin, and not to healthy one. In 2010, Harder et al. repeated the results of Ong et al. and Nomura et al., but at the same time researchers showed that immunoreactivity of psoriasin, hBD-2 and -3 in lesional and non-lesional $A D$ skin is enhanced in comparison to healthy controls $[8,44,45]$. Clearly, AD is not associated with a general induction defect of AMPs [38]. Moreover, no significant correlation between RNase 7, psoriasin, and hBD-2 and -3 secretion and cutaneous colonization by $S$. aureus nor with the severity of the disease was noticed [8]. These findings suggest another cause or a role of other AMPs in increased susceptibility of AD skin to S. aureus infections.

Interestingly, the expression of sweat gland-derived dermcidin was found to be decreased in AD patients [42]. Additionally, the inflammation in AD lesions is probably neutralizing the expected induction of human cathelicidin protein and even induces suppression [47]. In contrast, Ballardini et al. found a higher expression of cathelicidin LL-37 in lesional atopic skin compared with non-lesional atopic skin [48]. This might be in line with an observation that UVB phototherapy leads to healing of eczema lesions by an increased production of vitamin $D$, the same one that directly regulates the human cathelicidin expression in keratinocytes and induces LL-37 production in the skin in $\mathrm{AD}$ patients through oral supplementation [49].

Scratching as a result of itching, one of the main symptoms in AD, inevitably causes wounding of atopic skin. Injury in the case of patients with AD downregulates the expression of LL-37 [47]. On the other hand, a disrupted skin barrier cause enhanced immunoreactivity of hBD-2 and hBD-3 and strong secretion of RNase 7. Thus, Harder et al. has come to a conclusion that the disturbed skin barrier may trigger AMP induction in AD [8]. It is worth mentioning in regard to the disrupted skin barrier that extensive skin washing and the use of detergents may lead to a marked loss of AMPs, especially from lipid-rich regions or sebaceous glands [43].

Furthermore, even in severe AD no superinfection of the skin with $E$. coli was observed. It was revealed that the expression of psoriasin, a strong E. coli killing AMP, in non-lesional and lesional skin of untreated patients with $A D$ is increased in comparison to healthy controls [46]. Nevertheless, the role of AMPs in the pathogenesis of $A D$ is still unclear.

Some AMPs like hBD-2 and hBD-3 were primarily discovered in psoriasis. This chronic, inflammatory and hyperproliferative skin disease is associated with fewer superinfections of the skin than expected. Thus, this observation has led to a speculation that lesional psoriatic skin might produce antimicrobial peptides. Several studies acknowledged the theory $[13,16]$. Psoriasin, hBD-2 and hBD-3, LL-37, RNase 7 and calprotectin are induced in the psoriatic skin $[50,51]$. The up-regulation of AMPs expression in the skin of psoriasis patients is considered to contribute to a low rate of infections in psoriatic lesions. However, beside the antimicrobial function, AMPs play also immune modulatory functions in psoriasis. Especially the role of LL-37 as a modulator of psoriasis development has been highlighted in recent years. Interestingly, LL-37 presents a contrasting role in self-DNA mediated inflammation in the lesional psoriatic skin. Dermal cathelicidin LL-37 binds self-DNA and triggers cutaneous inflammation by activation of dermal plasmacytoid dendritic cells, whereas epidermal LL-37 joins with cytosolic DNA in keratinocytes and blocks its pro-inflammatory activity $[52,53]$. This might explain why vitamin $D_{3}$, a strong inducer of cathelicidin expression in keratinocytes and monocytes, is effective for psoriasis and reduces inflammation in psoriatic lesions [54].

Skin derived AMPs may play a role in the pathogenesis of other inflammatory diseases. Human $\beta$-defensins have been found in human pilosebaceous units, which are involved in the pathogenesis of acne vulgaris. Additionally, a marked increase in hBD-2 in lesional and perilesional epithelium of patients with acne vulgaris is observed, possibly as a consequence of inflammation [55]. Inflammation in acne is associated, in part, with abnormal ductal colonization of $P$. acnes. The bacterium induces the expression of AMPs which may contribute to the inflammation $[56,57]$. Nagy et al. demonstrated the upregulation of hBD-2 and IL-8 by genotypically $P$. acnes via TLR-2 and TLR-4 [57]. While hBD-2 serves as a chemoattractant, higher levels of the peptide may induce neutrophils and T cells migration to the site of infection and lead to inflammatory responses. According to Nagy et al., hBD-2 has no direct antimicrobial effects on $P$. acnes [57]. Thus, a higher expression of hBD-2 might not restrict the growth of the bacterium, unless it acts synergistically with human cathelicidin hCAP18/LL-37 [58]. On the contrary, LL-37 exhibits growth limiting ability against $P$. acnes. Like hBD-2, LL-37 is found in sebaceous glands. LL-37 presents both pro- and antiinflammatory properties and influences the function of TLR. This AMP is able to neutralize LPS. As a response to $P$. acnes there is an increased gene expression of LL-37 [59]. However, it has been suggested that killing 
potential of human cathelicidin might be significant in synergy with other AMPs like psoriasin [58]. Moreover, psoriasin is present in acne-involved sebaceous glands as well and upregulated in acne vulgaris lesions [60, 61]. Also RNase 7 shows in vitro the activity against $P$. acnes and is present in hair follicle epithelium [38]. Data on the significance of many AMPs in acne vulgaris are limited. Nevertheless, a synergistic effect of antimicrobial peptides against $P$. acnes and their potency in acne vulgaris pathogenesis have been suggested.

Rosacea is another chronic, inflammatory skin condition characterized by facial erythema, pustules and papules, and telangiectasia [62]. A specific cause for rosacea has not been identified, although symptoms are exacerbated by different factors and complex pathways are involved leading to inflammation and vascular response. Moreover, recent studies suggest a role of the skin innate immunity in the pathophysiology of rosacea. Triggering the innate immune system leads to changes in the expression of cytokines and AMPs like cathelicidin. Patients with rosacea express higher levels of cathelicidin in epidermis. Apart from the antimicrobial function, cathelicidin presents pro-inflammatory "alarmin" properties and affects angiogenesis. Furthermore, an increase in serine protease kallikrein 5 activity has been identified [63]. KLK5, also known as stratum corneum tryptic enzyme SCTE, is a processing enzyme of cathelicidin $[64,65]$. One of the forms of cathelicidin is LL-37, which appears to be generated in the epidermis of rosacea patients [66]. In addition, keratinocytes in the rosacea skin show a significantly higher TLR2 mRNA expression than in the normal skin [67]. Thus, the upregulated TLR2 level results in an abnormal expression and subsequent higher activity of KLK5, which in turn leads to altered levels of cathelicidin and possible induction of rosacea symptoms.

An altered expression of antimicrobial peptides is noticed also in other inflammatory skin diseases like acne inversa (hidradenitis suppurativa) and lichen sclerosus. Various studies have reported a significant increase in psoriasin, hBD-2 and LL-37 in both diseases [51, 68-70].

\section{Summary}

There is a growing number of studies suggesting a role of the skin innate immune system in the pathophysiology of inflammatory skin diseases. The inherent association of AMPs with inflammation has led to an assumption that dysregulated production of AMPs affects the development of chronic inflammatory diseases such as psoriasis, rosacea, acne vulgaris, or atopic dermatitis. AMPs actively contribute to host defense by mounting an innate immune response. They can exert a chemotactic influence on inflammatory cells. LL-37 acts as a chemoattractant for neutrophils, monocytes and T-cells. $\beta$-Defensins are chemotactic for immature dendritic cells and memory T-cells. Moreover, AMPs present ability to induce production of pro-inflammatory cytokines and chemokines. However, the primal role of antimicrobial peptides is to provide rapid, broad-spectrum defense against infection by acting as natural antibiotics. Alterations in the expression of AMPs are associated with increased susceptibility for skin colonization and/or infection by microbes. A lower effective concentration of AMPs allows for survival or proliferation of bacteria that would normally be eradicated. On the other hand, overexpression of AMPs in the skin may result in enhanced protection against skin infections as seen in patients with psoriasis and rosacea. In individuals with acne vulgaris, increased levels of AMPs are often found in the inflamed or infected skin areas indicating a role of these peptides in the protection from infection.

\section{Conflict of interest}

The authors declare no conflict of interest.

\section{References}

1. Erhart W, Alkasi Ö, Brunke G, et al. Induction of human beta-defensins and psoriasin in vulvovaginal human papillomavirus-associated lesions. J Infect Dis 2011; 204: 391-9.

2. Meyer-Hoffert U, Schwarz T, Schröder JM, Gläser R. Increased expression of human beta-defensin 3 in mollusca contagiosum. Clin Exp Dermatol 2010; 35: 190-2.

3. Meyer-Hoffert U, Schwarz T, Schröder JM, Gläser R. Expression of human beta-defensin-2 and -3 in verrucae vulgares and condylomata acuminata. J Eur Acad Dermatol Venereol 2008; 22: 1050-4

4. Nizet $\mathrm{V}$, Ohtake T, Lauth $\mathrm{X}$, et al. Innate antimicrobial peptide protects the skin from invasive bacterial infection. Nature 2001; 414: 454-7.

5. Ali RS, Falconer A, Ikram M, et al. Expression of the peptide antibiotics human beta defensin-1 and human beta defensin-2 in normal human skin. J Invest Dermatol 2001; 117: 106-11.

6. Rieg S, Garbe C, Sauer B, et al. Dermcidin is constitutively produced by eccrine sweat glands and is not induced in epidermal cells under inflammatory skin conditions. $\mathrm{Br}$ J Dermatol 2004; 151: 534-9.

7. Sørensen OE, Cowland JB, Theilgaard-Mönch K, et al. Wound healing and expression of antimicrobial peptides/polypeptides in human keratinocytes, a consequence of common growth factors. J Immunol 2003; 170: 5583-9.

8. Harder J, Dressel S, Wittersheim M, et al. Enhanced expression and secretion of antimicrobial peptides in atopic dermatitis and after superficial skin injury. J Invest Dermatol 2010; 130: 1355-64.

9. Lehrer RI, Ganz T. Defensins of vertebrate animals. Curr Opin Immunol 2002; 14: 96-102.

10. White SH, Wimley WC, Selsted ME. Structure, function, and membrane integration of defensins. Curr Opin Struct Biol 1995; 5: 521-7.

11. Kenshi Y, Gallo RL. Antimicrobial peptides in human skin disease. Eur J Dermatol 2008; 18: 11-21.

12. Ganz T, Selsted ME, Szklarek D, et al. Defensins. Natural peptide antibiotics of human neutrophils. J Clin Invest 1985; 76: 1427-35.

13. Harder J, Bartels J. A peptide antibiotic from human skin. Nature 1997; 387: 861. 
14. Schulz A, Klüver E, Schulz-Maronde S, Adermann K. Engineering disulfide bonds of the novel human beta-defensins hBD-27 and hBD-28: differences in disulfide formation and biological activity among human beta-defensins. Biopolymers 2005; 80: 34-49.

15. Liu AY, Destoumieux D, Wong AV, et al. Human beta-defensin-2 production in keratinocytes is regulated by interleukin-1, bacteria, and the state of differentiation. J Invest Dermatol 2002; 118: 275-81.

16. Harder J, Bartels J, Christophers E, Schröder JM. Isolation and characterization of human beta-defensin-3, a novel human inducible peptide antibiotic. J Biol Chem 2001; 276: 5707-13.

17. Schröder JM, Harder J. Human beta-defensin-2. Int J Biochem Cell Biol 1999; 31: 645-51.

18. Gläser R, Navid F, Schuller W, et al. UV-B radiation induces the expression of antimicrobial peptides in human keratinocytes in vitro and in vivo. J Allergy Clin Immunol 2009; 123: 1117-23.

19. Niyonsaba F, Ushio H, Nakano N, et al. Antimicrobial peptides human beta-defensins stimulate epidermal keratinocyte migration, proliferation and production of proinflammatory cytokines and chemokines. J Invest Dermat 2007; 127: 594-604.

20. Semple F, Webb S, Li HN, et al. Human beta-defensin 3 has immunosuppressive activity in vitro and in vivo. Eur J Immunol 2010; 40: 1073-8.

21. Yang D, Chertov O, Bykovskaia SN, et al. beta-Defensins: linking innate and adaptive immunity through dendritic and T cell CCR6. Science 1999; 286: 525-8.

22. Ferris LK, Mburu YK, Mathers AR, et al. Human beta defensin 3 induces maturation of human Langerhans cell like dendritic cells: an antimicrobial peptide that functions as an endogenous adjuvant. J Invest Dermatol 2013; 133: 460-8.

23. Oppenheim J, Biragyn A, Kwak L, Yang D. Roles of antimicrobial peptides such as defensins in innate and adaptive immunity. Ann Rheum Dis 2003; 62 (Suppl. 2): ii17-21.

24. Zanetti M, Gennaro R, Romeo D. Cathelicidins: a novel protein family with a common proregion and a variable Cterminal antimicrobial domain. FEBS Letters 1995; 374: 1-5.

25. Sørensen OE, Follin P, Johnsen AH, et al. Human cathelicidin, hCAP-18, is processed to the antimicrobial peptide LL-37 by extracellular cleavage with proteinase 3. Blood 2001; 97 : 3951-9.

26. Braff MH, Bardan A, Nizet V, Gallo RL. Cutaneous defense mechanisms by antimicrobial peptides. J Invest Dermatol 2005; 125: 9-13.

27. Dorschner RA, Pestonjamasp VK, Tamakuwala S, et al. Cutaneous injury induces the release of cathelicidin antimicrobial peptides active against group A Streptococcus. J Invest Dermatol 2001; 117: 91-7.

28. Koczulla R, von Degenfeld G, Kupatt C, et al. An angiogenic role for the human peptide antibiotic LL-37/hCAP-18. J Clin Invest 2003; 111: 1665-72.

29. Dombrowski Y, Schauber J. Cathelicidin LL-37: a defense molecule with a potential role in psoriasis pathogenesis. Exp Dermatol 2012; 21: 327-30.

30. De Yang, Chen O, Schmidt AP, et al. LL-37, the neutrophil granule- and epithelial cell-derived cathelicidin, utilizes formyl peptide receptor-like 1 (FPRL1) as a receptor to chemoattract human peripheral blood neutrophils, monocytes, and T cells. J Exp Med 2000; 192: 1069-74.

31. Madsen P, Rasmussen HH, Leffers H, et al. Molecular cloning, occurrence and expression of a novel partially secreted protein "psoriasin" that is highly up-regulated in psoriatic skin. J Invest Dermatol 1991; 97: 701-12.

32. Alowami S, Qing G, Emberley E, et al. Psoriasin (S100A7) expression is altered during skin tumorigenesis. BMC Dermatol 2003; 3: 1.

33. Gläser R, Harder J, Lange $H$, et al. Antimicrobial psoriasin (S100A7) protects human skin from Escherichia coli infection. Nature Immunol 2005; 6: 57-64

34. Jinquan T, Vorum H, Larsen CG, et al. Psoriasin: a novel chemotactic protein. J Invest Dermatol 1996; 107: 5-10.

35. Abtin A, Eckhart L, Gläser R, et al. The antimicrobial heterodimer S100A8/S100A9 (calprotectin) is upregulated by bacterial flagellin in human epidermal keratinocytes. J Invest Dermatol 2010; 130: 2423-30.

36. Thorey IS, Roth J, Regenbogen J, et al. The Ca2+-binding proteins S100A8 and S100A9 are encoded by novel injuryregulated genes. J Biol Chem 2001; 276: 35818-25.

37. Broome AM, Ryan D, Eckert RL. S100 protein subcellular localization during epidermal differentiation and psoriasis. J Histochem Cytochem 2003; 51: 675-85.

38. Simanski M, Köten B, Schröder JM, et al. Antimicrobial RNases in cutaneous defense. J Innate Immun 2012; 4: 241-7.

39. Zhang J, Dyer KD, Rosenberg HF. Human RNase 7: a new cationic ribonuclease of the RNase A superfamily. Nucleic Acids Res 2003; 31: 602-7.

40. Harder J, Schröder JM. RNase 7, a novel innate immune defense antimicrobial protein of healthy human skin. J Biol Chem 2002; 277: 46779-84.

41. Köten B, Simanski M, Gläser R, et al. RNase 7 contributes to the cutaneous defense against Enterococcus faecium. PLoS One 2009; 4: e6424.

42. Rieg S, Steffen H, Seeber S, et al. Deficiency of dermcidinderived antimicrobial peptides in sweat of patients with atopic dermatitis correlates with an impaired innate defense of human skin in vivo. J Immunol 2005; 174: 8003-10.

43. Harder J, Schröder JM, Gläser R. The skin surface as antimicrobial barrier: present concepts and future outlooks. Exp Dermatol 2013; 22: 1-5.

44. Nomura I, Goleva E, Howell MD, et al. Cytokine milieu of atopic dermatitis, as compared to psoriasis, skin prevents induction of innate immune response genes. J Immunol 2003; 171: 3262-9.

45. Ong PY, Ohtake T, Brandt C, et al. Endogenous antimicrobial peptides and skin infections in atopic dermatitis. New Engl J Med 2002; 347: 1151-60.

46. Gläser R, Meyer-Hoffert U, Harder J, et al. The antimicrobial protein psoriasin (S100A7) is upregulated in atopic dermatitis and after experimental skin barrier disruption. J Invest Dermatol 2008; 129: 641-9.

47. Mallbris L, Carlén L, Wei T, et al. Injury downregulates the expression of the human cathelicidin protein hCAP18/LL-37 in atopic dermatitis. Exp Dermatol 2010; 19: 442-9.

48. Ballardini N, Johansson C, Lilja G, et al. Enhanced expression of the antimicrobial peptide LL-37 in lesional skin of adults with atopic eczema. Br J Dermatol 2009; 161: 40-7.

49. Hata TR, Kotol P, Jackson M, et al. Administration of oral vitamin $\mathrm{D}$ induces cathelicidin production in atopic individuals. J Allergy Clin Immunol 2008; 122: 829-31.

50. Harder J, Schröder JM. Psoriatic scales: a promising source for the isolation of human skin-derived antimicrobial proteins. J Leukocyte Biol 2005; 77: 476-86.

51. Algermissen B, Sitzmann J, LeMotte P, Czarnetzki B. Differential expression of CRABP II, psoriasin and cytokeratin 1 mRNA in human skin diseases. Arch Dermatol Res 2005; 288: 426-30. 
52. Reinholz M, Ruzicka T, Schauber J. Cathelicidin LL-37: an antimicrobial peptide with a role in inflammatory skin disease. Ann Dermatol 2012; 24: 126-35.

53. Dombrowski Y, Peric M, Koglin S, et al. Cytosolic DNA triggers inflammasome activation in keratinocytes in psoriatic lesions. Sci Transl Med 2011; 3: 82ra38.

54. Morizane S, Gallo RL. Antimicrobial peptides in the pathogenesis of psoriasis. J Dermatol 2012; 39: 225-30.

55. Chronnell CMT, Ghali LR, Ali RS, et al. Human beta defensin- 1 and -2 expression in human pilosebaceous units: upregulation in acne vulgaris lesions. J Invest Dermatol 2001; 117: 1120-5.

56. Nagy I, Pivarcsi A, Koreck A, et al. Distinct strains of Propionibacterium acnes induce selective human beta-defensin-2 and interleukin-8 expression in human keratinocytes through Toll-like receptors. J Invest Dermatol 2005; 124 : 931-8.

57. Nagy I, Pivarcsi A, Kis K, et al. Propionibacterium acnes and lipopolysaccharide induce the expression of antimicrobial peptides and proinflammatory cytokines/chemokines in human sebocytes. Microbes Infect 2006; 8: 2195-205.

58. Lee DY, Yamasaki K, Rudsil J, et al. Sebocytes express functional cathelicidin antimicrobial peptides and can act to kill Propionibacterium acnes. J Invest Dermatol 2008; 128: 1863-6.

59. Lee SE, Kim JM, Jeong SK, et al. Protease-activated receptor-2 mediates the expression of inflammatory cytokines, antimicrobial peptides, and matrix metalloproteinases in keratinocytes in response to Propionibacterium acnes. Arch Dermatol Res 2010; 302: 745-56.

60. Borovaya A, Dombrowski Y, Zwicker S, et al. Isotretinoin therapy changes the expression of antimicrobial peptides in acne vulgaris. Arch Dermatol Res 2014; 306: 689-700.

61. Ganceviciene R, Fimmel S, Glass E, Zouboulis CC. Psoriasin and follicular hyperkeratinization in acne comedones. Dermatology (Basel) 2006; 213: 270-2.

62. Crawford GH, Pelle MT, James WD. Rosacea: I. Etiology, pathogenesis, and subtype classification. J Am Acad Dermatol 2004; 51: 327-41.

63. Yamasaki K, Di Nardo A, Bardan A, et al. Increased serine protease activity and cathelicidin promotes skin inflammation in rosacea. Nature Med 2007; 13: 975-80.

64. Yamasaki K, Gallo RL. The molecular pathology of rosacea. J Dermatol Sci 2009; 55: 77-81.

65. Yamasaki K, Schauber J, Coda A, et al. Kallikrein-mediated proteolysis regulates the antimicrobial effects of cathelicidins in skin. FASEB J 2006; 20: 2068-80.

66. Yamasaki K, Gallo RL. Rosacea as a disease of cathelicidins and skin innate immunity. J Invest Dermatol Symp P 2011; 15: $12-5$.

67. Yamasaki K, Kanada K, Macleod DT, et al. TLR2 expression is increased in rosacea and stimulates enhanced serine protease production by keratinocytes. J Invest Dermatol 2011; 131: 688-97.

68. Emelianov VU, Bechara FG, Gläser R, et al. Immunohistological pointers to a possible role for excessive cathelicidin (LL-37) expression by apocrine sweat glands in the pathogenesis of hidradenitis suppurativa/acne inversa. Br J Dermatol 2012; 166: 1023-34.

69. Schlapbach C, Yawalkar N, Hunger RE. Human beta-defensin-2 and psoriasin are overexpressed in lesions of acne inversa. J Am Acad Dermatol 2009; 61: 58-65.

70. Gambichler T, Skrygan M, Tigges C, et al. Significant upregulation of antimicrobial peptides and proteins in lichen sclerosus. Br J Dermatol 2009; 161: 1136-42. 